\title{
Microwave transmission properties of metamaterials with double sets of square holes
}

\author{
ZOU Si, LIU JinSong ${ }^{*} \&$ WANG KeJia \\ Wuhan National Laboratory for Optoelectronics, School of Optoelectronic Science and Engineering, Huazhong University of Science and \\ Technology, Wuhan 430074, China
}

Received February 10, 2012; accepted April 28, 2012; published online August 10, 2012

\begin{abstract}
The transmission property of microwave through a kind of metamaterial is investigated experimentally. Such metamaterials are fabricated with a structure of double sets of square holes: subwavelength ones, and small ones whose sizes and spacing are smaller at least one order of magnitude than those of the subwavelength holes. The experimental results show that the peak power of the measured transmission spectra is dependent on the structure parameters of the small holes. The physical origin to create this phenomenon is that the designer surface plasmons sustained by the small holes affect on the transmission property of the microwave passing through the subwavelength holes. The results are promising for proposing some techniques for optoelectronic devices in terahertz and microwave regime.
\end{abstract}

surface plasmons, surface waves, far infrared or terahertz, electromagnetic theory, guided waves, transmission

Citation: Zou S, Liu J S, Wang K J. Microwave transmission properties of metamaterials with double sets of square holes. Chin Sci Bull, 2012, 57: 3769-3772, doi: $10.1007 / \mathrm{s} 11434-012-5422-6$

A lot of works have been done in the topic of mimicking or spoof or designer surface plasmons (DSPs) on structured surfaces of a perfect conductor since this concept was presented by Pendry et al. [1,2]. The existence of DSPs was directly and indirectly verified in a microwave reflectivity measurement [3] and a terahertz enhanced transmission test [4], respectively. DSP propagation on structured metal surfaces was directly observed [5]. To understand the underlying mechanism of DSPs, some theoretical and experimental studies were presented recently [6-12]. Based on these fundamental results of DSPs, many interesting applications have been proposed, such as new-type terahertz collimators, waveguides, and filters [13-16].

The effect of DSP sates on the transmission property of electromagnetic waves through subwavelength apertures in metal films or foils at low frequency regime was studied theoretically if the metal-dielectric interface was structured [16]. A kind of metamaterial was proposed, in which an

*Corresponding author (email: jsliu4508@vip.sina.com) array of subwavelength apertures should be pierced into a metallic foil. One flat surface of the foil should be structured by making with periodic small indentations or holes with their size and spacing much smaller than those of the apertures [16]. Following the approach in [1,2], a theoretical model was build in [16], and it was demonstrated that the DSPs sustained by the small indentations or holes could affect the transmission property of the incident wave passing through the subwavelength apertures. The results from the model, which shows red-shift behavior of the transmission peaks, could be experimentally checked easily. If $\lambda_{\text {peak }}^{0}$ denotes the peak transmission wavelength when the interface is not structured (i.e. only pierced with arrays of subwavelength apertures), $\lambda_{\text {peak }}$ denotes that when the interface is structured by simultaneously pierced with an array of small indentations or holes, the red-shift behavior means $\lambda_{\text {peak }}>\lambda_{\text {peak }}^{0}$. Refer to the concept in [17], and this behavior can be named as transmission red-shift effect [16].

In order to verify the red-shift effect predicted in [16], a 
kind of metamaterials were made with a structure of double sets of circular holes: subwavelength ones and small ones. The diameter and spacing of the small holes are smaller at least one order of magnitude than those of the subwavelength ones. A microwave transmission experiment was done using these metamaterials [18]. However, the measured results were far away from the theoretical ones predicted in [16]. The main reason is that the theoretical model in [16] required that the depth of the holes should be deep enough, but the copper film of the experimental structure had a thickness three orders of magnitude smaller than the microwave wavelength. In order to overcome this problem, the theoretical model is modified by the effective dipoles theory [19]. The measured transmission peak wavelengths were in agreement with the modified theory [17]. Such measurements were performed only for the case of circular holes. We wondered what will happen if square holes are used. In this work, a set of metamaterials is made with double sets of square holes and their microwave transmission properties are measured. The observed results present a similar phenomenon as that for circular holes [18]. Unlike the case of circular holes, for the case of square holes it is difficult to obtain an analytical expression of the transmission peak wavelengths via the effective dipoles theory. As a result, we have to perform a numerical simulation using the finitedifference time domain (FDTD) method to directly solve Maxwell equations. The calculated transmission spectra are in agreement with the measured ones. The results not only demonstrate that the theoretical predication presented in [16] can be observed for the case of square holes, but also show that the red-shift amount depends on the geometrical parameters of the small holes.

The structure used in experiments, as schematically shown in the inset of Figure 1, is that a set of subwavelength square aperture with size $A$ arranged on a $D \times D$ lattice and such one square aperture is surrounded by square holes whose sizes $a$ and spacing $d$ are much smaller than $A$ and $D$. Using well-developed printed circuit board (PCB) technology, one can easily get such structures with different geometric parameters, one of which is shown in Figure 1. In

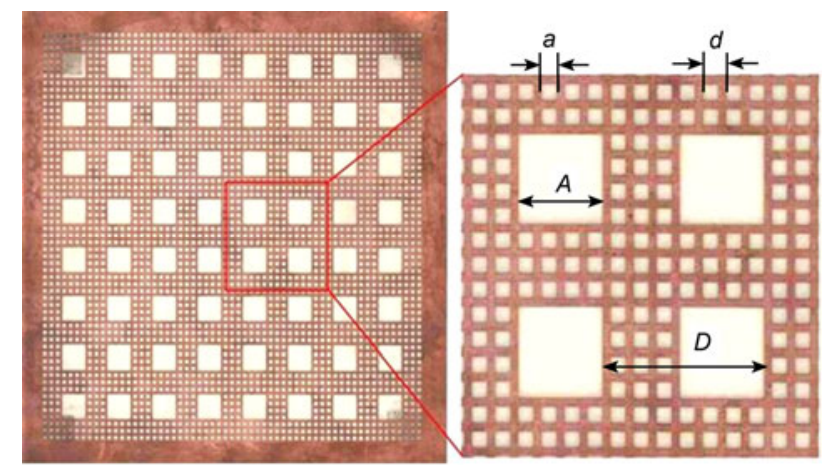

Figure 1 (Color online) One of fabricated samples used in experiments. Inset is the schematic of subwavelength apertures and the smaller square holes. order to demonstrate the conclusion that the transmission properties will be changed as the introduction of small square holes array; three groups of structures are fabricated. Each of group contains two samples: one is that only with subwavelength square aperture; the other has the same square aperture and smaller square holes. The geometric parameters of six samples are listed in the Table 1.

The transmission properties of all samples can be measured by two low sidelobe antennas. The incidence electromagnetic waves generated by one antenna, whose frequency covers from 7 to $16 \mathrm{GHz}$, are vertically impinged at the surface of samples, and the transmitted waves from the other surface can be detected by the receiving antenna. Using the Network Analyzer (N5230C, $10 \mathrm{MHz}-20 \mathrm{GHz}$, Agilent Tech.), we can visually observe the transmission distribution electromagnetic waves in the frequency domain.

The transmittance of two groups of samples, i.e. A0, A1, B0 and B1, as a function of frequency, is plotted in Figure 2(a) and (b), respectively. The black curves in each figure correspond to the samples only with subwavelength square aperture while the red curves are for the samples with square aperture and small square holes. As we can see from Figure 2(a) and (b), the EOT phenomenon is clearly illustrated for all of four samples. The existence of small holes arrays brings a red-shift of the transmittance curve shown in the Figure 2(a) and (b). For example, one can clearly see that although the shapes of two curves are almost same, the red one moves to the low frequency domain relative to the black one, as shown in Figure 2(a). The experimental result of the shift amount for samples A0 and A1 is approximately $\Delta f_{\mathrm{A}}^{\prime}=0.42 \mathrm{GHz}$ obtained from Figure 2(a). Similarly, the displacement between the transmission frequency peaks of samples $\mathrm{B} 0$ and $\mathrm{B} 1$ is $\Delta f_{\mathrm{B}}^{\prime}=0.018 \mathrm{GHz}$, as shown in the Figure 2(b). For the cases of A0, B0, and $\mathrm{C} 0$, there is no small hole arrays in them, and thus the EOT is due to the weakly confined surface modes, such as delocalized surface plasmons, instead of DSPs. On the other hand, DSPs sustained by the small holes are dominant in the EOT phenomenon for A1, B1, and C1. Particularly, the aperture arrays act like gratings, which induce the coupling between the external field in the air and DSPs supported by the small hole arrays. Based on this deference, the transmission peaks shift to the red for the samples with double sets of holes.

In order to theoretically investigate the transmission

Table 1 The geometric parameters of six samples for experiments

\begin{tabular}{ccccc}
\hline Sample & $A(\mathrm{~mm})$ & $D(\mathrm{~mm})$ & $a(\mathrm{~mm})$ & $d(\mathrm{~mm})$ \\
\hline A0 & 11 & 18 & & \\
A1 & 11 & 18 & 2 & 3 \\
B0 & 12 & 20 & & 5 \\
B1 & 12 & 20 & 2 & \\
C0 & 11 & 21 & & 3 \\
C1 & 11 & 21 & 2 & \\
\hline
\end{tabular}



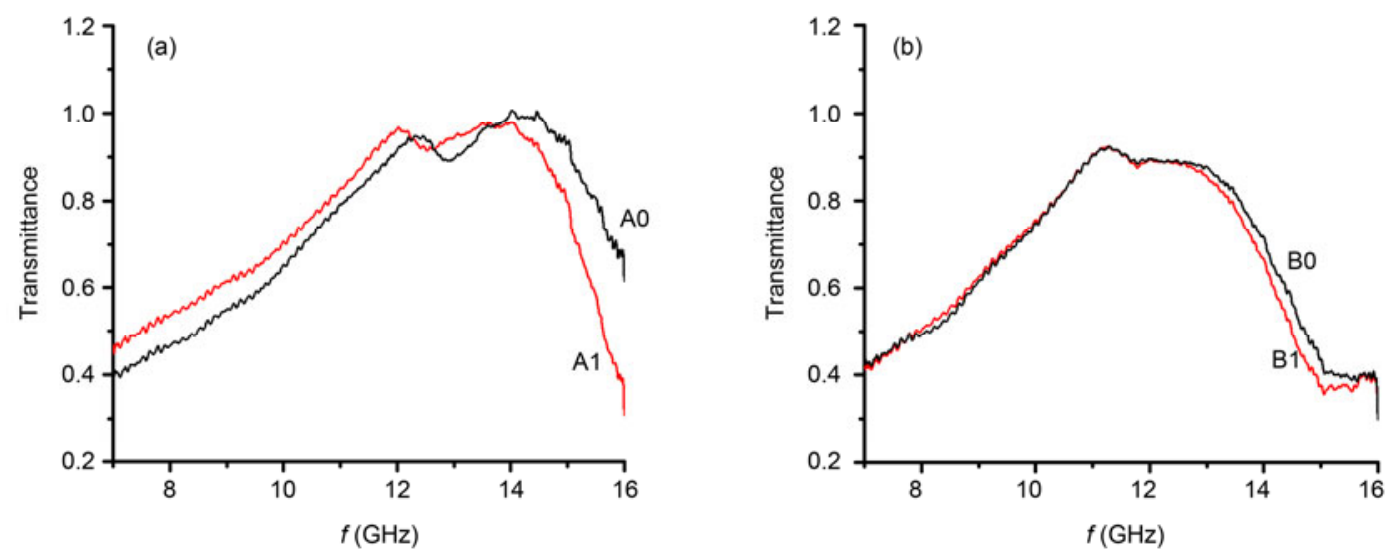

Figure 2 (Color online) The measured transmittance as a function of frequency for two groups of samples, respectively. (a) A0 and A1; (b) B0 and B1.

properties, we made a numerical simulation using the FDTD method to solve Maxwell equations directly, in which samples $\mathrm{C} 0$ and $\mathrm{C} 1$ are considered respectively. The experimental and simulation results for these two samples are illustrated in the Figure 3(a) and (b), respectively. Figure 3 shows that experimental and numerical results have a good agreement generally (the simulation for A0, A1, B0 and B1 is also performed, not shown in this paper). In other words, the deviation between experiment and simulation is very small. Firstly, transmittance curves from simulation, for $\mathrm{C} 0$ and $\mathrm{C}$, have almost the same profiles with these of the experiment. In addition, the main transmission peaks from the simulation for such two samples are 11.5 and $10.9 \mathrm{GHz}$, respectively, while the main peaks observed in experiment are 11.4 and $11.0 \mathrm{GHz}$. Further analysis of Figure 3(a) and (b) reveals that the shift amount obtained from simulation and experiments are 0.52 and $0.49 \mathrm{GHz}$, respectively.

It is also worthwhile to note that the shift amount has a certain relation to the numbers of smaller holes between two adjacent subwavelength square apertures, i.e. the amount will increase with growing of the number. The numbers, for

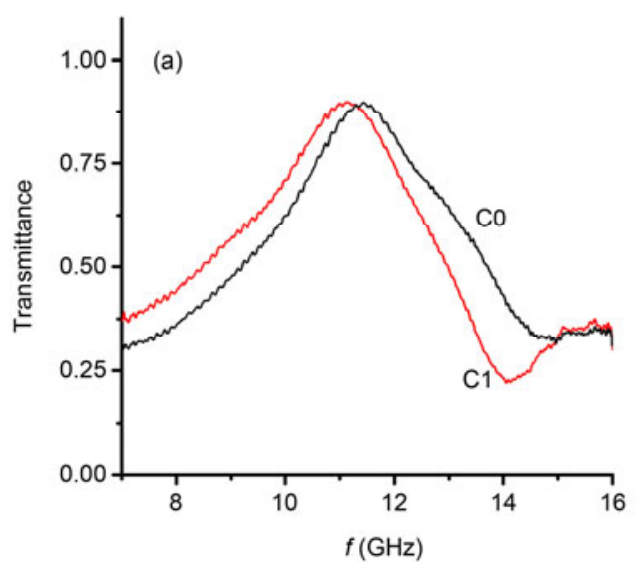

samples A1, B1 and C1, are 2, 1 and 3, respectively. The shift amounts for the three groups of samples, as mentioned before, are $0.42,0.018$ and $0.49 \mathrm{GHz}$, respectively. Thus, the transmission peak wavelength could be controlled by changing the numbers of smaller holes between two adjacent subwavelength square apertures. Moreover, through the experimental and simulation results, we also find that the positions of transmission peaks can be changed substantially and roughly by adjusting the aperture size and period, while they can be controlled slightly and precisely by changing the parameters of the small holes.

For a metamaterial with double sets of holes: subwavelength ones and small ones, it was revealed that the structure parameters of the small holes could affect on the transmission property of microwaves passing through the subwavelength holes for the case that the double sets of holes were circular. The results demonstrate that such phenomena can also be obtained when the double sets of holes are square. This study could enrich the possibilities to control the transmission properties of subwavelength structures via changing the geometric parameters of small holes arrays.

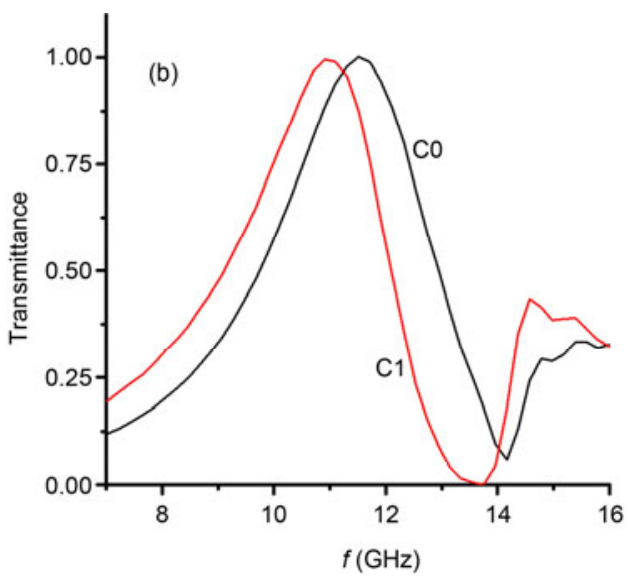

Figure 3 (Color online) The measured and simulation transmittance as a function of frequency for samples C0 and C1, respectively. (a) Measured results; (b) simulation results. 
This work was supported by the National Natural Science Foundation of China (10974063 and 61177095), Hubei Natural Science Foundation (2010CDA001), Ph.D. Programs Foundation of Ministry of Education of China (20100142110042) and the Fundamental Research Funds for the Central Universities (HUST: 2010MS041 and 2011TS001).

1 Pendry J B, Martin-Moreno L, Garcia-Vidal F J. Science, 2004, 305: 847-848

2 Garcia-Vidal F J, Martin-Moreno L, Pendry J B. J Opt A, 2005, 7: S97-S101

3 Hibbins A P, Evans B R, Sambles J R. Science, 2005, 308: 670-672

4 Tanaka M, Miyamaru F, Hangyo M, et al. Opt Lett, 2005, 30: 1210-1212

5 Williams C R, Andrews S R, Maier S A, et al. Nat Photon, 2008, 2: 175-179
6 Shen J T, Catrysse P B, Fan S H. Phys Rev Lett, 2005, 94: 197401

7 Smith D R, Pendry J B. J Opt Soc Am B, 2006, 23: 391-403

Shen L F, Chen X D. Opt Exp, 2008, 16: 3326-3333

9 Lan Y C, Chern R L. Opt Exp, 2006, 14: 11339-11347

10 Maier S A, Andrews S R. Appl Phys Lett, 2006, 88: 251120

11 García de Abajo F J, Sáenz J J. Phys Rev Lett, 2005, 95: 233901

12 Hendry E, Hibbins A P, Sambles J R. Phys Rev B, 2008, 78: 235426

13 Juluri B K, Lin S C S, Walker T R, et al. Opt Exp, 2009, 17: 2997-3006

14 Yu N F, Wang Q J, Kats M A, et al. Nat Mater, 2010, 9: 730-735

15 Talebi N, Shahabadi M. J Phys D: Appl Phys, 2010, 43: 135302

16 Liu J S, Ding L, Wang K J, et al. Opt Exp, 2009, 17: 12714-12722

17 García de Abajo F J, Gómez-Medina R, Sáenz J J. Phys Rev E, 2005, 72: 016608

18 Wang K J, Ding L, Liu J S, et al. Opt Exp, 2011, 19: 11375-11380

19 García de Abajo F J. Rev Mod Phys, 2007, 79: 1267-1290

Open Access This article is distributed under the terms of the Creative Commons Attribution License which permits any use, distribution, and reproduction in any medium, provided the original author(s) and source are credited. 